\title{
UNIQUE FACTORIZATION IN GRADED POWER SERIES RINGS
}

\author{
PETER S. LANDWEBER ${ }^{1}$
}

\begin{abstract}
It is shown that the graded ring $R\left[x_{1}, x_{2}, \cdots\right][[t]]$ of homogeneous power series is a graded UFD if $R$ is a regular UFD, the degrees of the $x_{i}$ are positive and tend to $\infty$, and $t$ has degree - 1. In particular this applies to $M U^{*}\left(C P^{\infty}\right)$ and $B P^{*}\left(C P^{\infty}\right)$.
\end{abstract}

1. Introduction. It is well known that if $R$ is a unique factorization domain (UFD) then so is any polynomial ring over $R$; on the other hand, P. Samuel [5] has shown that the power series ring $R[[t]]$ need not be a UFD. In the positive direction, if $R$ is a regular UFD then so is $R[[t]]$; in particular if $R$ is a principal ideal domain then $R\left[\left[x_{1}, \cdots, x_{n}\right]\right]$ is a UFD (see e.g. [1], [4] or [5]). At present it is apparently not known if $R\left[x_{1}, x_{2}, \cdots\right][[t]]$ is a UFD, even when $R$ is a field.

In this note we study unique factorization of homogeneous power series over graded rings. If $S=\left(S_{n}\right)_{n \in Z}$ is a graded ring, we call $s \in S_{n}$ a homogeneous element of $S$ of degree $n$, and write $|s|=n$. For a commutative graded ring $S$, let $S[[t]]$ denote the graded ring of homogeneous power series over $S$, where $|t|=-1$; thus $S[[t]]_{n}$ consists of power series $f=$ $\sum_{i=0}^{\infty} f_{i} t^{i}$ with $\left|f_{i}\right|=n+i$. Our main result, Theorem 3.1 , is that $S[[t]]$ is a graded UFD in case $S=R\left[x_{1}, x_{2}, \cdots\right]$ is a graded polynomial ring over a regular UFD $R$, where the degrees $\left|x_{i}\right|$ are positive and converge to $\infty$. We note that with this choice of gradings $R\left[x_{1}, x_{2}, \cdots\right][[t]]$ is isomorphic to the inverse limit of the graded rings $R\left[x_{1}, \cdots, x_{n}\right][[t]]$; in $\S 2$ we show that $R\left[x_{1}, \cdots, x_{n}\right][[t]]$ is a graded UFD (Corollary 2.3), and then pass to the limit in $\$ 3$.

In particular, it follows that $M U^{*}\left(C P^{\infty}\right)$ and $B P^{*}\left(C P^{\infty}\right)$ are graded UFD's, where $M U^{*}()$ denotes complex cobordism and $B P^{*}()$ is Brown-Peterson cohomology. (I apologize for using homology rather than cohomology indexing.) For $M U$ this result has been stated without proof and used by T. tom Dieck (see [2, p. 365] or [3, p. 34]); since the proof is deeper than one may first expect, it seems worthwhile to publish it.

Received by the editors January 31, 1973.

AMS (MOS) subject classifications (1970). Primary 13F15; Secondary 13J05, 55B20.

Key words and phrases. Unique factorization domain, graded ring, homogeneous power series.

1 Research supported in part by an NSF Grant 21064.

(c) American Mathematical Society 1974 
2. A reduction of the problem. Let $S$ be a graded integral domain, and $S[[t]],|t|=-1$, the graded ring of homogeneous power series over $S$.

(2.1) Proposition. $S[[t]]$ is a graded UFD if and only if the ring $S[[t]]\left[t^{-1}\right]_{0}$ is a UFD.

Notice that $S[[t]]\left[t^{-1}\right]_{0}$ is the ring of Laurent series $f=\sum_{i=n}^{\infty} f_{i} t^{i}$ with $f_{i} \in S_{i}$ and $n$ any integer. In particular, if the grading on $S$ is nonnegative then this coincides with $S[[t]]_{0}$, the ring of power series $f=\sum_{i=0}^{\infty} f_{i} t^{i}$ over $S$ with $\left|f_{i}\right|=i$.

Proof. Suppose that $S[[t]]$ is a UFD. Hence there is a set $\left\{p_{\alpha}\right\}$ of prime elements in $S[[t]]$ so that each nonzero $f \in S[[t]]$ has the form

$$
f=u t^{n_{0}} p_{\alpha_{1}}^{n_{1}} \cdots p_{\alpha_{r}}^{n_{r}}
$$

where $u$ is a unit and the exponents $n_{0}, \cdots, n_{r}$ are uniquely determined. It is immediate that $S[[t]]\left[t^{-1}\right]$ and $S[[t]]\left[t^{-1}\right]_{0}$ are UFD's, with a complete set of prime elements given by $\left\{p_{\alpha}^{\prime}\right\}$ where $p_{\alpha}^{\prime}=p_{x} t^{\left|p_{\alpha}\right|}$ has degree 0 .

Conversely, assume $S[[t]]\left[t^{-1}\right]_{0}$ is a UFD with $\left\{p_{\alpha}^{\prime}\right\}$ a complete set of prime elements. Choose $d_{\alpha}$ so that $p_{\alpha}=t^{-d_{\alpha}} p_{\alpha}^{\prime}$ belongs to $S[[t]]$ and has nonzero constant term (note that $d_{\alpha}=\left|p_{\alpha}\right|$ ). One checks easily that the $p_{\alpha}$ are prime elements in $S[[t]]$, and that each of its nonzero homogeneous elements in a product of the form (2.2). Thus $S[[t]]$ is a graded UFD.

(2.3) CoROllary. If $S=R\left[x_{1}, \cdots, x_{n}\right]$ is a graded polynomial ring, where $\left|x_{i}\right|>0$ and $R$ is a regular UFD, then $S[[t]]$ is a graded UFD.

Proof. Since $S$ is nonnegatively graded, $S[[t]]\left[t^{-1}\right]$ coincides with $S[[t]]_{0}$. In turn we may identify $S[[t]]_{0}$ with $R\left[\left[y_{1}, \cdots, y_{n}\right]\right]$, where $y_{i}=x_{i} t^{\left|x_{i}\right|}$, which is known to be a UFD (see e.g. [4, Theorem 188]).

3. The main theorem. In this section we fix a regular UFD $R$ and let $S$ denote the graded polynomial ring $R\left[x_{1}, x_{2}, \cdots\right]$ where $\left|x_{i}\right|>0$ and $\left|x_{i}\right| \rightarrow \infty$.

(3.1) TheOREM. $R\left[x_{1}, x_{2}, \cdots\right][[t]]$ is a graded UFD provided that $\left|x_{i}\right|>0,\left|x_{i}\right| \rightarrow \infty$ and $|t|=-1$.

Proof. For convenience we let $S_{n}$ denote the graded polynomial ring $R\left[x_{1}, \cdots, x_{n}\right]$. We define homomorphisms of $R$-algebras $\phi_{n}: S \rightarrow S_{n}$ and $\phi_{n}^{N}: S_{N} \rightarrow S_{n}$ for $N \geqq n$ by $x_{i} \mapsto x_{i}$ if $i \leqq n$ and $x_{i} \mapsto 0$ if $i>n$.

These induce homomorphisms

$$
\phi_{n}: S[[t]] \rightarrow S_{n}[[t]], \quad \phi_{n}^{N}: S_{N}[[t]] \rightarrow S_{n}[[t]], \quad N \geqq n
$$

by acting on the coefficients of the power series. In view of our assumption that $\left|x_{i}\right|>0$ and $\left|x_{i}\right| \rightarrow \infty$, it is immediate that the $\phi_{n}$ define an isomorphism

$$
S[[t]] \cong \underset{n}{\lim } S_{n}[[t]]
$$


of graded rings. We know each $S_{n}[[t]]$ is a graded UFD, and shall show how to pass to the limit.

To show that $S[[t]]$ is a graded UFD, it suffices to verify the following statements:

(3.2) Each nonzero element is a product of irreducible elements.

(3.3) Each irreducible element is prime.

We recall that $f$ is irreducible if $f=g h \Rightarrow g$ or $h$ is a unit; and $f$ is prime if $f|g h \Rightarrow f| g$ or $f \mid h$. A prime element is always irreducible; the converse holds in a UFD.

It suffices to establish (3.2) and (3.3) for homogeneous power series $f=\sum_{i=0}^{\infty} f_{i} t^{i}$ with $f_{0} \neq 0$, since $t$ is evidently a prime element.

As to (3.2), notice that, if $f=g h$ where $f=\sum t_{i} t^{i}\left(f_{0} \neq 0\right), g=\sum g_{i} t^{i}$ and $h=\sum h_{i} t^{i}$, then $f_{0}=g_{0} h_{0}$. Since $R\left[x_{1}, x_{2}, \cdots\right]$ is a UFD, we can easily express $f$ as a product of irreducible elements.

Prior to showing that irreducible elements are prime in $S[[t]]$, we verify the statement:

(3.4) If $f \in S[[t]]$ is irreducible, then for large $n$ also $\phi_{n}(f) \in S_{n}[[t]]$ is irreducible.

So let $f=\sum f_{i} t^{i}\left(f_{0} \neq 0\right)$ be irreducible and write $f_{0}=p_{1} \cdots p_{s}$ where the $p_{i}$ are irreducible in $R\left[x_{1}, x_{2}, \cdots\right]$. Choose $N$ so that $n>N \Rightarrow\left|x_{n}\right|>\left|f_{0}\right|$; then for $n \geqq N$ also $\phi_{n}(f)$ has constant coefficient $f_{0}$. Thus $\phi_{n}(f)$ is a product of $r_{n}$ irreducible elements with $r_{n} \leqq s$; evidently we have $r_{n} \leqq r_{n+1} \leqq$ $s$, so if we increase $N$ sufficiently then $n \geqq N \Rightarrow r_{n}=n$ is independent of $n$. Hence we may assume that for $n \geqq N$ we have

$$
\phi_{n}(f)=p_{1}^{(n)} \cdots p_{n}^{(n)}
$$

where the $p_{i}^{(n)}$ are irreducible elements of $S_{n}[[t]]$.

We next show that it is possible to arrange that

$$
\phi_{n}^{n+1}\left(p_{i}^{(n+1)}\right)=p_{i}^{(n)}, \quad n \geqq N .
$$

To see this, begin with $\phi_{N}(f)=p_{1}^{(N)} \cdots p_{r}^{(N)}$ and continue by induction on $n$. Thus if we have achieved $\phi_{n}(f)=p_{1}^{(n)} \cdots p_{r}^{(n)}$ we first choose the $p_{i}^{(n+1)}$ provisionally so that

$$
\phi_{n+1}(f)=p_{1}^{(n+1)} \cdots p_{r}^{(n+1)} .
$$

Applying $\phi_{n}^{n+1}$, we may permute the $p_{i}^{(n+1)}$ and obtain $\phi_{n}^{n+1}\left(p_{i}^{(n+1)}\right)=u_{i} p_{i}^{(n)}$ with $u_{i}$ a unit. If we replace $p_{i}^{(n+1)}$ by $p_{i}^{(n+1)} u_{i}^{-1}$ for $i<r$, then we have

$$
\phi_{n}^{n+1}\left(p_{i}^{(n+1)}\right)=p_{i}^{(n)} \text { for } i<r ;
$$

from $\phi_{n}(f)=\phi_{n}^{n+1}\left(\phi_{n+1}(f)\right)$ we see that also $\phi_{n}^{n+1}\left(p_{r}^{(n+1)}\right)=p_{r}^{(n)}$. This establishes (3.6). 
Thus for $i=1, \cdots, r$ the elements $\left\{p_{i}^{(n)}\right\}_{n \geqq N}$ determine an element of the inverse limit of the $S_{n}[[t]]$, hence we obtain $p_{1}, \cdots, p_{r}$ in $S[[t]]$ so that $\phi_{n}\left(p_{i}\right)=p_{i}^{(n)}$ for $n \geqq N$. This gives $\phi_{n}(f)=\phi_{n}\left(p_{1} \cdots p_{r}\right)$ for $n \geqq N$, so $f=p_{1} \cdots p_{r}$. No $p_{i}$ is a unit and $f$ is irreducible, hence $r=1$ and we have verified (3.4).

We are ready for (3.3). Let $f$ be irreducible in $S[[t]]$ and assume $f \mid g h$. For large $n$ we know $\phi_{n}(f)$ is prime, hence $\phi_{n}(f)$ divides $\phi_{n}(g)$ or $\phi_{n}(h)$. Thus we may suppose that $\phi_{n}(f)$ divides $\phi_{n}(g)$ for all $n$. We see immediately that $f$ divides $g$, as desired. So $f$ is a prime element. Q.E.D.

(3.7) Remark. One easily recognizes as irreducible any power series in $R\left[x_{1}, x_{2}, \cdots\right][[t]]$ whose constant term is an irreducible element of $R\left[x_{1}, x_{2}, \cdots\right]$. This covers the power series that arise in [2] and [3]. It is easy to show that such an irreducible element is prime, in both the graded and ungraded case; the point is that the analogue of (3.4) is now automatic.

(3.8) REMARK. In view of (2.1), we have shown that the power series ring $R\left[\left[x_{1}, x_{2}, \cdots\right]\right]$ over a regular UFD is a UFD if $\left|x_{i}\right|>0$ and $\left|x_{i}\right| \rightarrow \infty$. Its elements have the form $f=\sum_{i=0}^{\infty} f_{i}$ where $f_{i} \in R\left[x_{1}, x_{2}, \cdots\right]_{i}$ is a polynomial of degree $i$.

(3.9) RemarK. One measure of the difficulty in the ungraded case is that we only obtain a monomorphism of $R\left[x_{1}, x_{2}, \cdots\right][[t]]$ into the inverse limit of the $R\left[x_{1}, \cdots, x_{n}\right][[t]]$, hence we cannot prove the analogue of 3.4 by our argument.

\section{REFERENCES}

1. D. A. Buchsbaum, Some remarks on factorization in power series rings, J. Math. Mech. 10 (1961), 749-753. MR 23 \#A1666.

2. T. tom Dieck, Actions of finite abelian p-groups without stationary points, Topology 9 (1970), 359-366. MR 44 \#2253.

3. Kobordismentheorie klassifizierender Räume und Transformations gruppen, Math. Z. 126 (1972), 31-39.

4. I. Kaplansky, Commutative rings, Allyn and Bacon, Boston, Mass., 1970. MR 40 \#234.

5. P. Samuel, Lectures on unique factorization domains, Tata Institute of Fundamental Research Lectures on Math., no. 30, Tata Institute of Fundamental Research, Bombay, 1964. MR 35 \#5428.

Department of Mathematics, Rutgers University, New Brunswick, New JERSEY 08903 